\title{
Five Unique Temperate Phages from a Polylysogenic Strain of Bacillus thuringiensis Subsp. aizawai
}

\author{
By ROBERT B. REYNOLDS, $\uparrow$ AMALA REDDY $\ddagger$ AND \\ CURTIS B. THORNE* \\ Department of Microbiology, University of Massachusetts, Amherst, Massachusetts 01003, USA
}

(Received 16 November 1987; revised 28 January 1988)

Five temperate phages were isolated from strain 4042B of Bacillus thuringiensis subsp. aizawai. The phages, which were heteroimmune, could also be distinguished by their host ranges, plaque and particle morphologies, serological specificities, and locations of restriction endonuclease cleavage sites on their chromosomes. Besides maintaining a stable lysogenic relationship with the 4042B host strain, each phage formed a stable lysogen with Bacillus cereus.

\section{INTRODUCTION}

Strains of Bacillus thuringiensis have elicited a special interest because they produce a proteinaceous parasporal crystal that is toxic to susceptible insect larvae. Since this commercially important species has only limited means of genetic exchange, temperate phages could be important genetic tools, serving as mediators of generalized and specialized transduction and as potential cloning vehicles. We have thus been interested in the characterization of temperate phages from this species.

Past surveys of strains of $B$. thuringiensis have suggested that a significant proportion harbour one or more temperate phages (Colasito \& Rogoff, 1969; Ackermann \& Smirnoff, 1978; Jones et al., 1983). However, the physical characteristics of these phages, as well as their relationships with the host strains, have not been examined in detail. Several phages that mediate generalized transduction in B. thuringiensis (Thorne, 1978; Landen et al., 1981; Heierson et al., 1983) and one that converts spore-negative, crystal-negative cells to spore-positive, crystal-positive cells (Perlak et al., 1979) have been described. More recently we have utilized transducing phages to link genetic markers on the $B$. thuringiensis chromosome (Barsomian et al., 1984) and to transfer plasmids among strains of $B$. thuringiensis, $B$. cereus and $B$. anthracis (Ruhfel et al., 1984).

During our investigation of strain 4042B of $B$. thuringiensis subsp. aizawai, we isolated five unique temperate phages whose characteristics are described in this report. The phages are heteroimmune and they can be distinguished on the basis of plaque and particle morphology, host range, antigenicity and restriction endonuclease digestion patterns of their DNAs.

\section{METHODS}

Bacterial strains. Derivatives of $B$. thuringiensis $4042 B$ and other strains that were used for detection or propagation of phages are listed in Table 1. Strains that were used only for the determination of phage host ranges are included in Table 2.

Bacteriophages. TP-15, TP-16, TP-17, TP-19 and TP-20 were isolated from $B$. thuringiensis $4042 \mathrm{~B}$ as described in Results. CP-55 was isolated from a culture filtrate of B. cereus 13472 . TP-15c, TP-16c, TP-17c, TP-20c and TP-19v were spontaneous clear-plaque (c) or virulent (v) mutants.

† Present address: Henkel Research Corporation, 2330 Circadian Way, Santa Rosa, CA 95407, USA. $\ddagger$ Present address: Department of Biological Sciences, Wellesley College, Wellesley, MA 02181, USA.

Abbreviation: MC, mitomycin C. 


$\quad$ Strain
Bacillus thuringiensis
4042B
4042B UM50
Bacillus cereus
4415
4415 UM1
4415 UM1(TP-19)
4415 UM1(TP-20)
13472
13472 UM1
13472 UM8
13472(TP-15)
13472(TP-16)
13472(TP-17)
13472 UM1(TP-17)

\section{Table 1. List of strains}

Characteristics

Subsp. aizawai

trp-2

Wild-type

trp-1

trp-1, carries TP-19

trp-1, carries TP-20

Wild-type, carries CP-55

thr-1

ile-1

Carries TP-15

Carries TP-16

Carries TP-17

thr-1, carries TP-17

\section{Origin/reference}

Barsomian et al. (1984)

UV mutagenesis of 4042B

ATCC
UV mutagenesis of 4415
This study
This study
ATCC
UV mutagenesis of 13472
UV mutagenesis of 13472
This study
This study
This study
This study

Media and growth conditions. L broth, PA broth and agar, and peptone diluent were prepared as described previously (Thorne, 1978). BHI-L broth was made by adding $12 \mathrm{ml}$ sterile double strength Difco brain-heart infusion broth (BHI) to $88 \mathrm{ml}$ sterile L broth. CAY broth contained $12 \mathrm{~g} \mathrm{~N}-\mathrm{Z}$ Case Peptone (Sheffield Chemical Co.) and $3 \mathrm{~g}$ Difco yeast extract per litre. Difco nutrient broth ( $\mathrm{N}$ broth) and $\mathrm{BHI}$ broth were prepared according to instructions from the manufacturer. $\mathrm{N}$ agar and soft $\mathrm{N}$ agar were prepared by adding 15 and $5 \mathrm{~g}$ Difco agar, respectively, per litre of $\mathrm{N}$ broth. Cultures were incubated at $37^{\circ} \mathrm{C}$ on a rotary shaker $(250$ r.p.m.) unless otherwise described. Spores were prepared from cultures grown on potato agar as described (Thorne, 1962).

Propagation and assay of phages. TP-15 and TP-15c were propagated on cells of $B$. cereus 13472 in soft N agar overlays on $\mathbf{N}$ agar plates. The overlays were seeded with phage from a plaque suspended in $1 \%(\mathrm{w} / \mathrm{v})$ peptone. TP16 and TP-16c were propagated from phage lysates by mixing $1 \times 10^{9}$ p.f.u. and approximately $3 \times 10^{8}$ spores in 3 $\mathrm{ml}$ soft $\mathrm{N}$ agar and overlaying on $\mathrm{N}$ agar plates. Overlay plates were incubated for $20 \mathrm{~h}$ and the soft agar layer was suspended in $6 \mathrm{ml} \mathrm{N}$ broth. TP-17 and TP-17c were grown by lytic infection of L broth cultures of B. cereus 13472 UM1. Three to five plaques of phage were suspended in $1 \mathrm{ml} 1 \%$ peptone and added to $25 \mathrm{ml}$ culture in the exponential phase of growth. Following removal of debris by slow speed centrifugation, phage lysates were filtered through Millipore DA membranes.

TP-19 and TP-20 were propagated by induction of their $B$. cereus 4415 UM1 lysogens during exponential growth with mitomycin $\mathrm{C}(\mathrm{MC})$ at $0.7 \mu \mathrm{g} \mathrm{ml}^{-1}$ final concentration. The $\mathrm{MC}$ was removed after $15 \mathrm{~min}$ by pelleting the cells and resuspending them in $25 \mathrm{ml}$ fresh BHI broth. TP-19v and TP-20c were propagated from plaques on cells of $B$. cereus 4415 in soft PA agar overlays on PA agar plates. Overlays were incubated $16 \mathrm{~h}$ before the phage was harvested as described for TP-15 and TP-16.

To induce $B$. cereus 13472 lysogens carrying TP-15 or TP-16, a $10 \%(\mathrm{v} / \mathrm{v})$ transfer of a $12 \mathrm{~h} \mathrm{~L}$ broth culture was made into BHI-L broth. After $135 \mathrm{~min}, 10 \mathrm{ml}$ was transferred to $15 \mathrm{ml}$ BHI-L broth containing MC at a final concentration of $0.4 \mu \mathrm{g} \mathrm{ml}^{-1}$. Induction of $B$. cereus $13472 \mathrm{UM1}$ (TP-17) was by the same procedure except that CAY broth was substituted for BHI-L broth.

Lysates of TP-17, TP-19, TP-20 and CP-55 were stored at $15^{\circ} \mathrm{C}$ after filtration and those of TP-15 and TP-16 were stored at $4^{\circ} \mathrm{C}$.

All phages were assayed by the soft agar overlay technique (Adams, 1959). TP-15 and TP-16 and their clearplaque mutants were assayed in $N$ agar with $B$. cereus 13472 spores as the indicator. Spores of $B$. cereus 13472 were also used for TP-17 and TP-17c but PA agar was substituted for N agar. TP-19, TP-19v, TP-20, TP-20c and CP-55 were assayed with $B$. cereus $\mathbf{4 4 1 5}$ cells as the indicator in PA agar.

Purification of phages. Phage lysates were incubated with DNAase $\left(1 \mu \mathrm{g} \mathrm{m}^{-1}\right)$ for $30 \mathrm{~min}$ at $37^{\circ} \mathrm{C}$. The phages were concentrated by centrifugation and purified in $\mathrm{CsCl}$ density gradients as described previously (Perlak et al., 1979). TP-17, TP-19, TP-20 and CP-55 were centrifuged at $15^{\circ} \mathrm{C}$, and the other phages were centrifuged at $4{ }^{\circ} \mathrm{C}$.

Phage antiserum. Purified phage suspensions were homogenized with an equal volume of Freund's incomplete adjuvant (Difco) and injected into rabbits. Each rabbit received $3 \mathrm{ml}$ intramuscularly and $1 \mathrm{ml}$ subcutaneously. Rabbits were bled after 5 weeks and serum was heated at $56^{\circ} \mathrm{C}$ for $30 \mathrm{~min}$. The neutralization rates $(K$ values) were determined by the procedure of Eisenstark (1967).

Electron microscopy. Purified phage stocks were negatively stained with $2 \%(\mathrm{w} / \mathrm{v})$ aqueous uranyl acetate, $2 \%$ (w/v) sodium phosphotungstate or $2 \%(w / v)$ ammonium molybdate, $\mathrm{pH} 7 \cdot 0$, on carbon-coated grids and examined in an RCA EMU-4 or Siemens 102A electron microscope.

Isolation and analysis of DNA. The procedure of isolating DNA from purified phage stocks was described previously (Perlak et al., 1979). Phage DNAs were digested by restriction endonucleases according to procedures from BRL. Lambda DNA was obtained from BRL. DNA fragments were electrophoresed in horizontal $1 \%(w / v)$ 
Table 2. Host ranges of the five phages

Bacterial strain

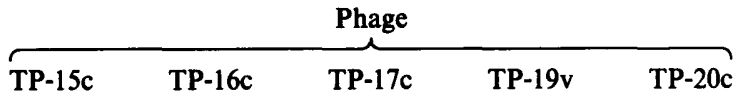

B. thuringiensis

NRRL ${ }^{*}$ B-4040, subsp. finitimus

NRRL B-4041, subsp. alest $i$

NRRL B-4043, subsp. dendrolimus

NRRL B-4044, subsp. kenyae

NRRL B-4047, subsp. entomocidus-limassol

NRRL B-4049, subsp. morrisoni

NRRL, B-4050, subsp. tolworthi

NRRL B-4055, subsp. kurstaki

NRRL B-4058, subsp. darmstadiensis

NRRL B-4059, subsp. toumanoffi

4042A, subsp. thuringiensist

4042B, subsp. aizawai ${ }^{\dagger}$

BIS, subsp. israelensis $\ddagger$

B. cereus

NRRL B-569

ATCC 4415

ATCC 7064

ATCC 9139

ATCC 9592

ATCC 9620

ATCC 13472

$\begin{array}{lll}+ & - & - \\ + & - & - \\ + & - & + \\ - & - & - \\ + & - & - \\ - & + & - \\ - & - & - \\ + & + & + \\ - & - & - \\ + & + & + \\ - & - & + \\ - & - & - \\ + & + & + \\ + & - & + \\ + & - & + \\ - & - & + \\ + & + & + \\ + & - & + \\ + & + & +\end{array}$

+ , lysis of test strain; -, no lysis of test strain.

* NRRL, Agricultural Research Service, Northern Regional Research Laboratory, US Department of Agriculture, Peoria, Illinois, USA.

+ Barsomian et al. (1984).

¥ Obtained from H. de Barjac.

or $0.7 \%$ agarose gels containing Tris/borate buffer $(0.089 \mathrm{M}$-Trizma base, $0.089 \mathrm{M}$-boric acid and $0.0025 \mathrm{M}$-EDTA, $\mathrm{pH} 8.3)$. The gels were stained with ethidium bromide $\left(1.5 \mu \mathrm{g} \mathrm{ml}^{-1}\right)$ and photographed over a short wave UV light source.

\section{RESULTS}

\section{Isolation of the phages}

When filtrates of $B$. thuringiensis $4042 B$ cultures were assayed with $B$. cereus 13472 as indicator, three different pure phage clones were isolated, designated TP-15, TP-16, and TP- 17 . Each of the phages formed morphologically distinct turbid plaques. Subsequently, two new types of morphologically distinct turbid plaques were observed when filtrates were obtained in the presence of antisera to the three known phages and assayed on B. cereus 4415 . These phages were named TP-19 and TP-20.

After repeated single plaque isolations, lysates of the different plaque types still contained a low proportion of clear-plaque-forming units. These spontaneous clear-plaque or virulent mutants of TP-15, TP-16, TP-17, TP-19 and TP-20 were used for the determination of host ranges. Purified suspensions of TP-15c, TP-16c and TP-17c were also used for physical characterization of the different phage types. Propagation of these clear-plaque mutants by lytic infection of $B$. cereus 13472 produced the large quantities of phages required for the purification procedure and also avoided the problem of significant contamination of lysates with the resident CP-55 phage that is carried by strain 13472. TP-19 and TP-20 phages for the purification procedure were obtained by MC induction of their respective B. cereus 4415 UM1 lysogens isolated from colony-centred plaques.

Host ranges, particle morphologies and antigenic uniqueness

Table 2 shows that each of the five phage types had a unique host range when tested with strains of $B$. thuringiensis and $B$. cereus. B. cereus 13472 was chosen as the host for routine assays 

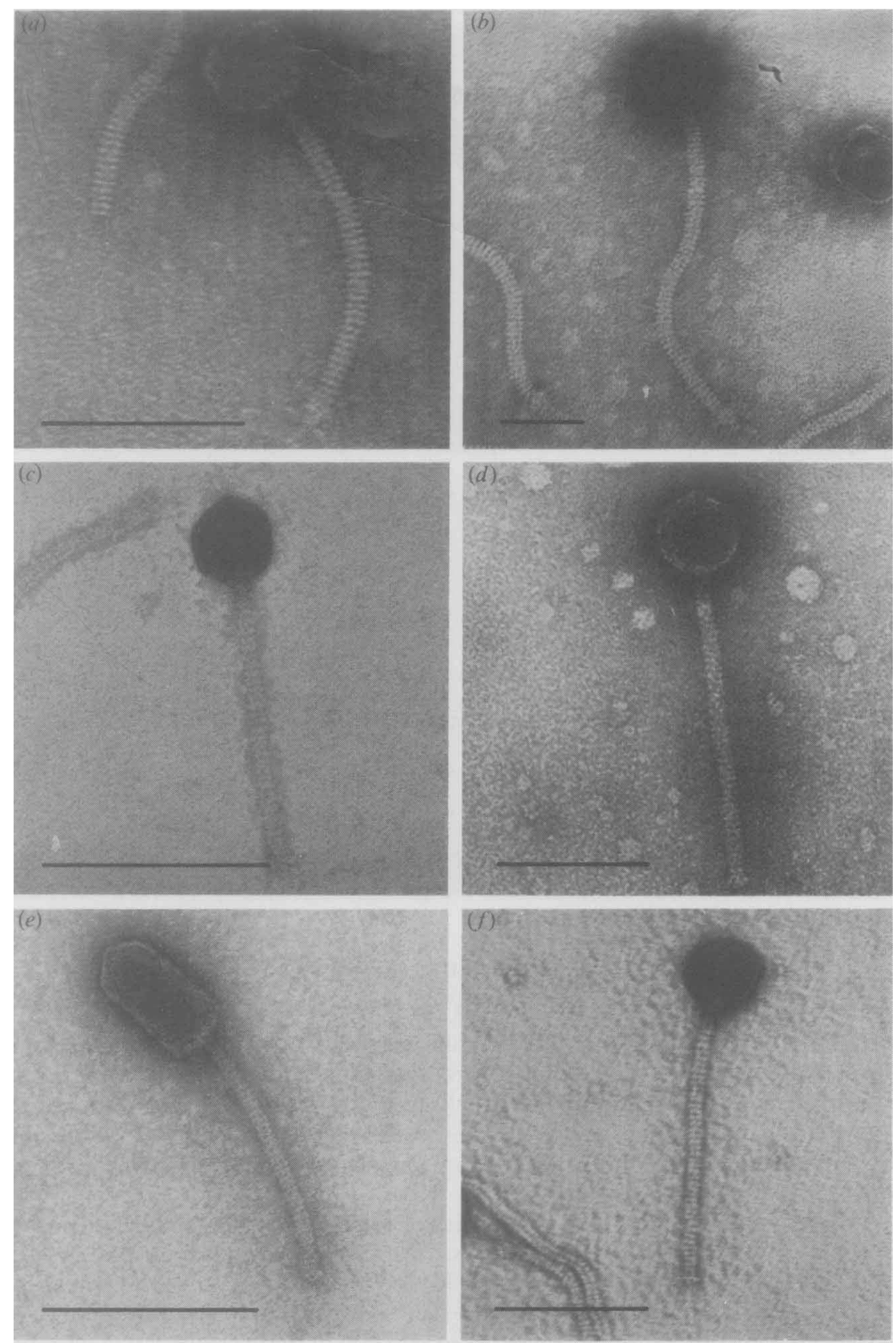

Fig. 1. Electron micrographs of (a) TP-15c, (b) TP-16c, (c) TP-17c, (d) TP-19, (e) TP-20 and $(f)$ CP-55 stained with uranyl acetate. The bar represents $100 \mathrm{~nm}$ in $(a),(c),(d),(e)$ and $(f)$ and $60 \mathrm{~nm}$ in $(b)$.

and propagation of TP-15, TP-16 and TP- 17 because that strain gave more distinct plaques and higher efficiencies of plating than any of the other sensitive strains tested. For the same reasons $B$. cereus 4415 was used for assay and propagation of TP-19 and TP-20.

With the exception of TP-20, the phages isolated from $B$. thuringiensis 4042B had similar particle morphologies (Fig. $1 a-e$ ) and dimensions (Table 3). Phage tails were flexible and 
Table 3. Dimensions of phage particles

Values are means of measurements of at least 15 particles. SD values are given in parentheses.

$\begin{array}{lcc}\text { Phage } & \begin{array}{c}\text { Head diameter } \\ (\mathrm{nm})\end{array} & \begin{array}{c}\text { Tail length } \\ (\mathrm{nm})\end{array} \\ \text { TP-15c } & 51(1 \cdot 6) & 184(6 \cdot 2) \\ \text { TP-16c } & 53(1 \cdot 6) & 248(6 \cdot 3) \\ \text { TP-17c } & 47(1 \cdot 3) & 135(7 \cdot 3) \\ \text { TP-19 } & 48(1 \cdot 6) & 170(7 \cdot 1) \\ \text { TP-20 } & 35(0.5) \times 70(2 \cdot 4) & 128(3 \cdot 3)\end{array}$

Table 4. Neutralization of phages by antisera

\begin{tabular}{crrrrr} 
Phage & \multicolumn{5}{c}{$K$ value for phage } \\
antiserum & TP-15c & TP-16c & TP-17c & TP-19 & TP-20 \\
TP-15c & 261000 & 67 & 2 & 3 & 3 \\
TP-16c & 49 & 497000 & 2 & 3 & 3 \\
TP-17c & 3 & 17 & 2710 & 4 & 3 \\
TP-19 & 2 & 1 & 1 & 164 & 27 \\
TP-20 & 2 & 1 & 1 & 2 & 186
\end{tabular}

noncontractile. Four or five short projections were visible at the ends of TP-15c and TP-16c tails when the particles were stained with sodium phosphotungstate (not shown).

The $K$ value of a phage antiserum is a measure of its neutralizing potency, a high $K$ value indicating a high titre. Thus, the $K$ values obtained when a particular antiserum is tested against different phages are indicative of the degrees of serological relatedness of the phages (Adams, 1959). As shown in Table 4, the $K$ values indicate that each of the five phages was serologically unrelated to the others.

Under the described conditions less than one active CP-55 particle per $10^{3}$ p.f.u. was present in the $B$. thuringiensis phage lysates (data not shown). CP-55 had a negligible effect on the determination of TP-15c, TP-16c, and TP-17c host ranges in Table 2 since it was able to infect only the $B$. cereus 4415 strain of those listed. The relatively low proportions of CP-55 virions in lysates of TP-15c, TP-16c and TP-17c did not significantly affect the physical characterization of these phages. Examination of numerous fields of stained TP-15c, TP-16c and TP-17c particles did not reveal any virions resembling CP-55 (Fig. $1 f$ ), but CP-55-like particles could be found among phage purified from a filtrate of an MC-induced culture of $B$. cereus 13472 (TP-16). Dilutions of TP-15c-, TP-16c- or TP-17c-specific antiserum which completely neutralized the homologous phage did not significantly neutralize CP-55 activity (data not shown).

\section{Phage DNAs}

Comparison of fragments from EcoRI and HindIII endonuclease digests of DNA extracted from purified TP-15c, TP-16c, TP-17c, TP-19 and TP-20 particles indicated that the different phage chromosomes were physically unique (Table 5). The EcoRI-cut fragments of TP-15c, TP$16 \mathrm{c}$ and TP-17c DNA were not the same size as those from an EcoRI digest of CP-55 DNA thus demonstrating that only insignificant amounts of CP-55 DNA could have been present in those preparations. None of the B. thuringiensis phage DNAs could be digested by BamHI, and TP17c DNA was not cut by HindIII.

The sizes of the EcoRI and HindIII fragments in Table 5 were estimated by comparing their relative migration distances in agarose gels with those of fragments from EcoRI, HindIII and EcoRI-BamHI digests of lambda DNA. The size of each phage DNA, as determined from the sum of the sizes of its DNA fragments, is given in Table 5. 
Table 5. Restriction fragments of phage DNAs

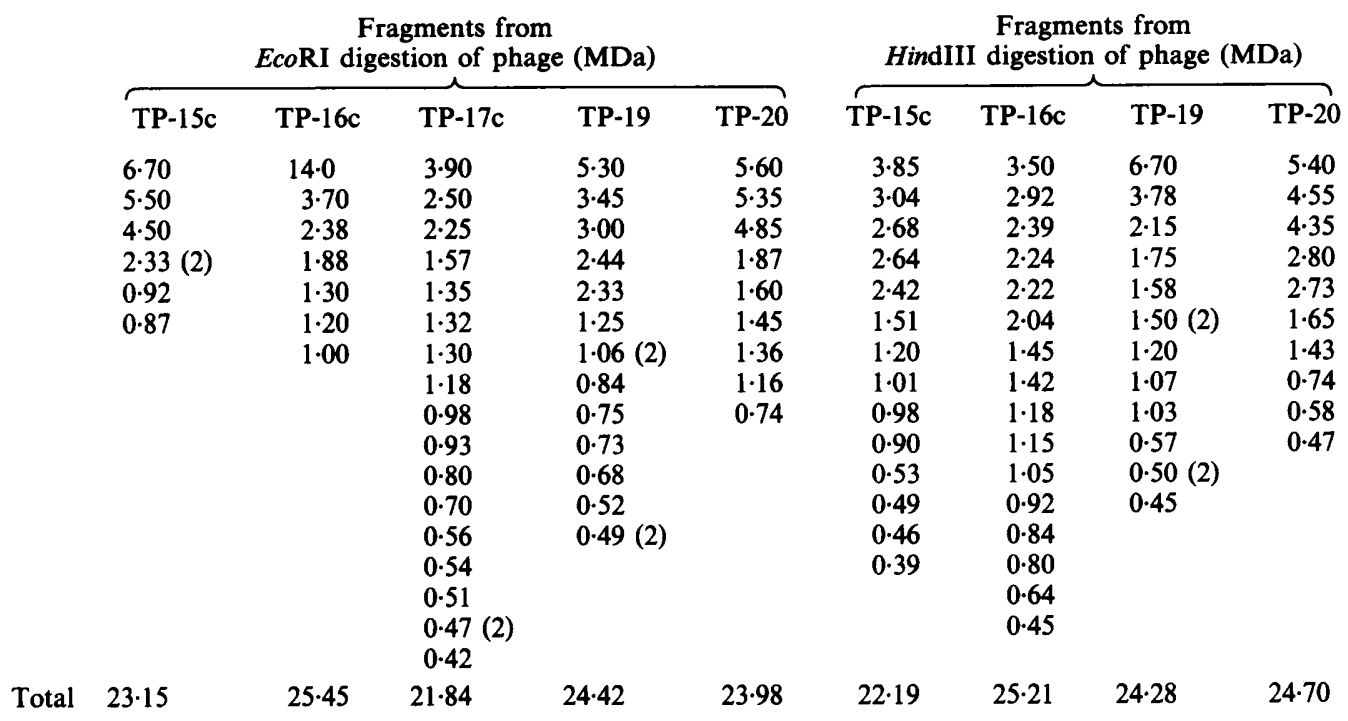

Table 6. Sensitivity of B. cereus lysogens to the five phages

Sensitivity of strains was tested in phage assays and in cross-streaks.

$\begin{array}{lccccc}\text { Strain tested } & \text { TP-15c } & \text { TP-16c } & \text { TP-17c } & \text { TP-19 } & \text { TP-20c } \\ \text { 13472 } & + & + & + & + & \text { Whage } \\ \text { 4415 UM1 } & + & \text { W } & + & + & + \\ \text { 13472(TP-15) } & - & + & + & + & \text { W } \\ \text { 13472(TP-16) } & + & - & + & + & \text { W } \\ \text { 13472(TP-17) } & - & - & - & + & \text { W } \\ \text { 4415 UM1(TP-19) } & - & \text { W } & + & - & + \\ \text { 4415 UM1(TP-20) } & + & \text { W } & + & + & - \\ + \text {, Strain was sensitive; } & - \text {, strain was not sensitive; W, wild-type strain was not sensitive. }\end{array}$

\section{Lysogenizing characteristics of the phages}

Stable $B$. cereus lysogens of each of the five phages isolated from $B$. thuringiensis 4042B were obtained by isolation from the centres of turbid plaques. Attempts to cure such lysogens, as well as $B$. thuringiensis $4042 \mathrm{~B}$, by repeated single colony isolations were unsuccessful. All of the five phages were reisolated from 4042B UM50 after the strain was serially subcloned by single colony isolation for a total of 21 times. Furthermore, all five phages were reisolated from strain 4042B UM50 spores prepared by allowing a PA broth culture to sporulate in the presence of sufficient phage-specific antiserum to completely neutralize all plaque-forming activity and then heating the resulting spores at $80^{\circ} \mathrm{C}$ for $30 \mathrm{~min}$.

From the results shown in Table 6, it appears that each of the phages possessed a distinctive immunity, although TP-16 and TP-20 immunities could not be compared in this experiment due to their mutually exclusive host ranges. Although TP-15c was unable to lyse the TP-19 lysogen, TP-19 could lyse the TP-15 lysogen. Similarly, neither TP-15c nor TP-16c could lyse the TP-17 lysogen, but TP-17c could lyse TP-15 and TP-16 lysogens. Strain 4042B was immune to each of the five phages and their clear-plaque mutants but was lysed by the virulent mutant of phage TP-19. 


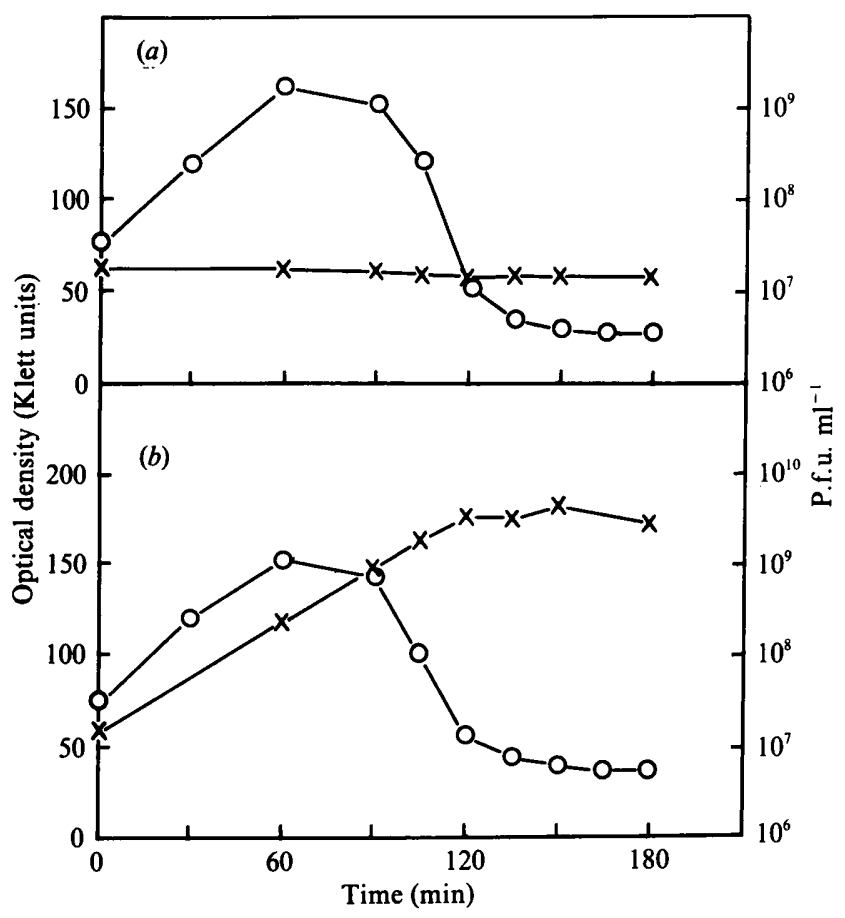

Fig. 2. MC induction of (a) B. thuringiensis 4042B UM50 and (b) B. cereus 13472(TP-16). Each strain was induced by the procedure described for $B$. cereus 13472 (TP-16), i.e. the culture was transferred at 0 min to broth containing MC (see Methods). Optical density (given in Klett units) was determined with a Klett-Summerson colorimeter equipped with a red (no. 66) filter. O, Optical density; X, TP-16 titre.

Table 7. Phage yields following mitomycin $C$ induction of $B$. cereus lysogens

\begin{tabular}{lcc}
\multicolumn{1}{c}{ Strain } & $\begin{array}{c}\text { Untreated culture } \\
\left(\text { p.f.u. } \mathrm{ml}^{-1}\right)\end{array}$ & $\begin{array}{c}\text { MC-induced culture } \\
(\text { p.f.u. ml }\end{array}$ \\
13472(TP-15) & $4.85 \times 10^{7}$ & $4.60 \times 10^{9}$ \\
$13472($ TP-16) & $6.61 \times 10^{7}$ & $1.09 \times 10^{10}$ \\
$13472($ TP-17) & $6.00 \times 10^{1}$ & $1.03 \times 10^{3}$ \\
4415 UM1(TP-19) & $2.00 \times 10^{6}$ & $7.20 \times 10^{9}$ \\
4415 UM1(TP-20) & $1.80 \times 10^{6}$ & $4.00 \times 10^{9}$
\end{tabular}

Treatment of lysogenized Bacillus strains with DNA-damaging agents such as MC or UV light will often greatly increase the rate of induction and release of phage particles (Warner et al., 1977). Treatment of $B$. thuringiensis $4042 B$ cultures with $M C$ at several different concentrations caused massive culture lysis but unexpectedly did not bring about any significant increase in the titres of the five phages (data not shown). In an example of this phenomenon (Fig. 2a), an MCtreated culture of strain 4042B did not release an increased amount of TP-16 during lysis. However, MC treatment of a culture of the $B$. cereus 13472 (TP-16) lysogen under the same conditions did significantly increase the TP-16 titre (Fig. $2 b$ ). Table 7 shows that $B$. cereus lysogens prepared from each of the five phages isolated from $B$. thuringiensis $4042 B$ released increased amounts of phage upon induction with MC.

\section{Transduction}

Each of the five phages was examined for the ability to mediate generalized transduction. Mutants of $B$. cereus $\mathbf{1 3 4 7 2}$ or $\mathbf{4 4 1 5}$ were used as recipients for phage grown on wild-type strains. TP-17 was the only one of the five phages able to effect generalized transduction. The $t h r-1$ and 
ile-1 markers of strain 13472 were transduced at an average frequency of $6 \times 10^{-7}$ transductants per p.f.u. Other markers were not tested. TP-17 was temperate enough so that inactivation of p.f.u. was not necessary for survival of transductants.

\section{Plasmid profiles of lysogens}

A comparison of plasmid profiles of $B$. thuringiensis 4042B, B. cereus strains 13472 and 4415 , and $B$. cereus lysogens carrying the five phages indicated there were no new plasmids in the lysogens. Four plasmids with estimated molecular masses of 5.0, 9.0, 10.2 and >100 MDa were present in 4042B UM50. Hybridization experiments with TP-15c and TP-16c DNA probes confirmed a lack of homology with any of the 4042B plasmids (Reynolds, 1982). Similar hybridization experiments using DNA from the three other phages as probes were not done.

\section{DISCUSSION}

$B$. thuringiensis 4042B is a polylysogen that is able to maintain a stable relationship with five different prophages. Although Jones et al. (1983) isolated 11 possibly temperate phages in a survey of 36 strains of the aizawai subspecies, no strain carrying more than one phage was identified. Other investigators have previously identified $B$. thuringiensis strains that carry more than one phage (Ackermann \& Smirnoff, 1978).

Previous investigations of temperate phages inhabiting $B$. subtilis have placed them into four or five major groups based on similar antigenicity, homoimmunity and phage adsorption sites (Dean et al., 1978). Phages within several of these groups share extensive (>80\%) DNA homology according to endonuclease analysis and heteroduplex mapping (Rudinski \& Dean, 1979). Even the dissimilar SPO2 and $\phi 105$ temperate phages share $14 \%$ DNA homology and are serologically related (Chow et al., 1972). In contrast to these examples, the five temperate phages carried by strain $4042 B$ were unrelated by every criterion examined. The uniqueness of the TP-15c, 16c and 17c genomes demonstrated by restriction endonuclease analysis was confirmed by Southern blotting experiments (Reynolds, 1982). Heterogeneity, in at least some functions (e.g. immunity), is most likely essential for maintenance of different temperate phages in a single host.

The absence of new plasmids in lysogens of $B$. cereus carrying the five phages suggests that the five phages integrate into the host chromosome. Assuming that the mass of the $B$. thuringiensis chromosome is equivalent to that estimated for the chromosome of $B$. subtilis, i.e. $2.0 \times 10^{9}-2.5$ $\times 10^{9} \mathrm{Da}$ (Henner \& Hoch, 1980), the integrated prophages would amount to a total of 4.8 to $6 \%$ of the 4042B chromosome.

The results presented here suggest that when the prophages in strain 4042B are simultaneously induced by treatment with MC, they interfere with each other's induction or replication (Fig. 2). Of the five phages carried by strain 4042B, TP-17 was notable for its strikingly low titres $(1 \times$ $10^{3}$ or fewer p.f.u. $\mathrm{ml}^{-1}$ ) released spontaneously or by $\mathrm{MC}$-induced cultures of $\mathrm{B}$. thuringiensis 4042B and the B. cereus 13472 (TP-17) lysogen. Each of the strains in question harbours one or more additional heteroimmune phages which may have somehow interfered with TP-17 induction and/or replication. Also, the inability of TP15c or TP-16c to lyse the $B$. cereus 13472(TP-17) lysogen and of TP-15c to lyse the 4415 UM1(TP-19) lysogen (Table 6) suggests that several of the phages carried by strain 4042B have specific mechanisms of interference with phage infection or replication.

Based on the size of its genome (21.8 MDa; Table 5) and its ability to mediate generalized transduction, TP-17 may be valuable for fine mapping of linked chromosomal markers in $B$. thuringiensis and $B$. cereus.

While characterizing TP-16, we isolated a spontaneous deletion mutant which we designated TP-16-1. Restriction endonuclease digestions of TP-16 and 16-1 DNAs showed that TP-16-1 has about $1.13 \mathrm{MDa}$ DNA deleted from the adjacent 1.3 and 2.38 MDa EcoRI fragments of TP-16. Since this deletion of TP-16 DNA did not affect phage immunity or its essential replication functions, insertion of foreign DNA into this region may form a nondefective specialized 
transducing phage. This method may prove useful for the construction of cells diploid for the $B$. thuringiensis crystal protein gene and hence, it may result in the improvement of $B$. thuringiensis strains for agricultural use.

We thank Christopher Woodcock for taking the electron micrographs of TP-17 and TP-20 and Judith A. Siess for her assistance in preparing the manuscript.

This work was supported in part by Grant PCM79-21851 from the National Science Foundation.

\section{REFERENCES}

ACKermanN, H. W. \& Smirnoff, W. A. (1978). Recherches sur la lysogenie chez Bacillus thuringiensis et B. cereus. Canadian Journal of Microbiology 24, 818-826.

ADAMs, M. H. (1959). Bacteriophages, pp. 450-451 New York, London \& Sydney: Wiley Interscience.

Barsomian, G. D., Robillard, N. J. \& Thorne, C. B. (1984). Chromosomal mapping of Bacillus thuringiensis by transduction. Journal of Bacteriology 157 , 746-750.

Chow, L. T., BoICE, L. B. \& DAvidson, N. (1972). Map of the partial sequence homology between DNA molecules of Bacillus subtilis bacteriophage SPO2 and $\phi 105$. Journal of Molecular Biology 68, 391-400.

Colasito, D. J. \& RogofF, M. H. (1969). Characterization of temperate bacteriophages of Bacillus thuringiensis. Journal of General Virology 5, 275-281.

DeAN, D. H., ForT, C. L. \& HoCH, J. A. (1978). Characterization of temperate phages of Bacillus subtilis. Current Microbiology 1, 213-217.

EISENSTARK, A. (1967). Bacteriophage techniques. Methods in Virology 1, 449-524.

Heierson, A., Landen, R. \& Boman, H. G. (1983). Transductional mapping of nine linked chromosomal genes in Bacillus thuringiensis. Molecular and General Genetics 192, 118-123.

Henner, D. J. \& HoCH, J. A. (1980). The Bacillus subtilis chromosome. Microbiological Reviews 44, 57 82.

Jones, D. R., Karunakaran, V. \& Burges, H. D. (1983). Phages naturally associated with the aizawai variety of insect pathogen Bacillus thuringiensis and their relevance to strain identification. Journal of Applied Bacteriology 54, 373-377.

Landen, R., Heierson, A. \& Boman, H. G. (1981). A phage for generalized transduction in Bacillus thuringiensis and mapping of four genes for antibiotic resistance. Journal of General Microbiology 123, 4959.

Perlak, F. J., Mendelsohn, C. L. \& Thorne, C. B. (1979). Converting bacteriophage for sporulation and crystal formation in Bacillus thuringiensis. Journal of Bacteriology 140, 699-706.

REYNOLDS, R. B. (1982). Characterization of temperate bacteriophages of a Bacillus thuringiensis strain. $\mathrm{PhD}$ dissertation, University of Massachusetts, USA.

RudinsKi, M. S. \& DEAN, D. H. (1979). Evolutionary considerations of related Bacillus subtilis temperate phage $\phi 105, \rho 14, \rho 10$ and $\rho 6$ as revealed by heteroduplex analysis. Virology 99, 57-66.

Ruhfel, R. E., Robillard, N. J. \& ThORNe, C. B. (1984). Interspecies transduction of plasmids among Bacillus anthracis, $B$. cereus, and $B$. thuringiensis. Journal of Bacteriology 157, 708-711.

THORNE, C. B. (1962). Transduction in Bacillus subtilis. Journal of Bacteriology 83, 106-111.

THORNE, C. B. (1978). Transduction in Bacillus thuringiensis. Applied and Environmental Microbiology 35, 1109-1115.

Warner, F. D., Kitos, G. A., Romano, M. P. \& HeMPHILL, H. E. (1977). Characterization of SP $\beta$ : a temperate bacteriophage from Bacillus subtilis 168M. Canadian Journal of Microbiology 23, 45-51. 